\title{
ESTUDO NUMÉRICO DO MAPA PADRÃO DISSIPATIVO RELATIVÍSTICO
}

\author{
Ana C.C. Horstmann, Holokx A. Albuquerque, Cesar Manchein
}

\author{
Departamento de Física, Universidade do Estado de Santa Catarina \\ Rua Paulo Malschitzki, s/N - Bairro Zona Industrial Norte - 89219-170 - JoinVille - SC - Brasil \\ E-MAIL: CAROLHORSTMANNQ YAHOO. COM. BR
}

\begin{abstract}
In this paper we study the dynamics of the standard map, in the conservative, dissipative and dissipative relativistic regimes, through a numerical approach. For the three cases above, were built their phase spaces, for through them, we can observe the existence and organization of periodic and chaotic domains. We also investigate the influence of the term in the relativistic dynamics of relativistic dissipative standard map and perform an analysis of how dissipation changes the structure of the phase space and quasi-periodic orbits.
\end{abstract}

Keywords - phase space, chaotic dynamics, standard map.

Resumo - Neste trabalho apresentamos os resultados obtidos da investigação da dinâmica do mapa padrão nas formas, conservativa, dissipativa e dissipativa relativística, através de uma abordagem numérica. Para os três casos supracitados, foram construídos e inspecionados os respectivos espaços de fases, pelos quais podemos constatar a coexistência de domínios caóticos e periódicos. Caracterizamos e discutimos ainda a influência dos parâmetros responsáveis pelo amortecimento ou dissipação e por uma componente relativística na dinâmica de uma partícula submetida a pulsos periódicos.

Palavras-chave — espaço de fases, dinâmica caótica, mapa padrão.

\section{Introdução}

Desde os trabalhos pioneiros de Chirikov, (1969) e Chirikov, (1979), quando propôs o mapa padrão, que descreve a dinâmica do rotor pulsado (ou quicado), vários trabalhos têm sido feitos na teoria dos sistemas Hamiltonianos. As principais vantagens em se estudar o mapa padrão, estão na riqueza de informações a respeito da dinâmica apresentada em seu espaço de fases, que podem ser estendidas a outros modelos. Tal motivo faz com que o mapa padrão de Chirikov seja um dos modelos mais estudados da literatura relacionada: pois ele pode ser aplicado em diferentes campos de Física, conforme pode ser constatado nas referências (Ciubotariu et al., 2002; Martins e Gallas, 2008; Oliveira et al., 2011 e Oliveira et al., 2012; Lan e Yapp, 2008), entre outras. Em outras palavras, este mapa pode ser usado para modelar o comportamento de vários sistemas físicos, como por exemplo: o rotor pulsado, o pêndulo forçado sem atrito, uma partícula em um campo eletromagnético e confinamento de plasma em tokamaks. Podemos ressaltar ainda que um fator comum em todos os problemas supracitados refere-se a importância da compreensão das principais propriedades inerentes à dinâmica dos mesmos.

Conforme citado no parágrafo anterior o mapa padrão pode ser obtido através do rotor pulsado, composto por uma haste de comprimento $L$ e momento de inércia $I$, fixada em uma das extremidades por um pino sem atrito, em torno do qual ela rotaciona e cujo movimento pode ser descrito em termos do momento e da posição angulares. O rotor pulsado vem sendo estudado por mais de 40 anos, devido essen- cialmente à possibilidade que existe em se estudar propriedades fundamentais referentes a dinâmica de sistemas, cuja energia é uma quantidade conservada ou não. A dinâmica deste tipo de sistemas ainda não está totalmente compreendida, e assim, almeja-se entender (e reconhecer) padrões ou estruturas em seu espaço de fases.

Neste trabalho temos como principais objetivos estudar a dinâmica do mapa padrão, em três formas diferentes: $(i)$ conservativa (quando a energia mecânica do sistema é uma quantidade conservada); (ii) dissipativa (quando energia mecânica for dissipada no tempo) e (iii) o mapa padrão relativístico quando adicionada uma certa quantidade de dissipação. Para tanto, analisaremos os espaços de fases, de forma sistemática, a medida que aumentarmos o valor do parâmetro que controla a dissipação em cada sistema. Neste cenário, esperamos que a dissipação altere bruscamente a dinâmica do regime em questão, especialmente com o surgimento de atratores no espaço de fases, conforme observado previamente por Martins e Gallas (2008) e suas referências.

Este artigo está organizado da seguinte maneira: na Seção 2 apresentaremos uma breve dedução do mapa padrão conservativo a partir do rotor pulsado e apresentaremos alguns espaços de fases para este caso, bem como a sua descrição. Na Seção 3 introduziremos o mapa padrão dissipativo e alguns resultados numéricos para este caso para diferentes valores do parâmetro de dissipação. Por outro lado na Seção 4, apresentaremos o mapa padrão dissipativo relativístico e os resultados numéricos referentes a investigação deste caso. Finalmente, na Seção 5 serão apre- 
sentadas as conclusões em seguida as referências bibliográficas.

\section{Mapa Padrão Conservativo}

Nesta seção temos como objetivo apresentar uma breve dedução do mapa padrão a partir do rotor pulsado. O rotor pulsado é essencialmente composto por uma barra rígida de comprimento $L$ e momento de inércia $I$, sendo uma das extremidades fixada por um pino sem atrito. Essa barra rotaciona em torno do pino, sem a influência da gravidade, com frequência constante ao mesmo tempo em que recebe pulsos (ou quique) periódicos de intensidade $K / L$ e período $\tau$, conforme mostrado na Fig. 1.

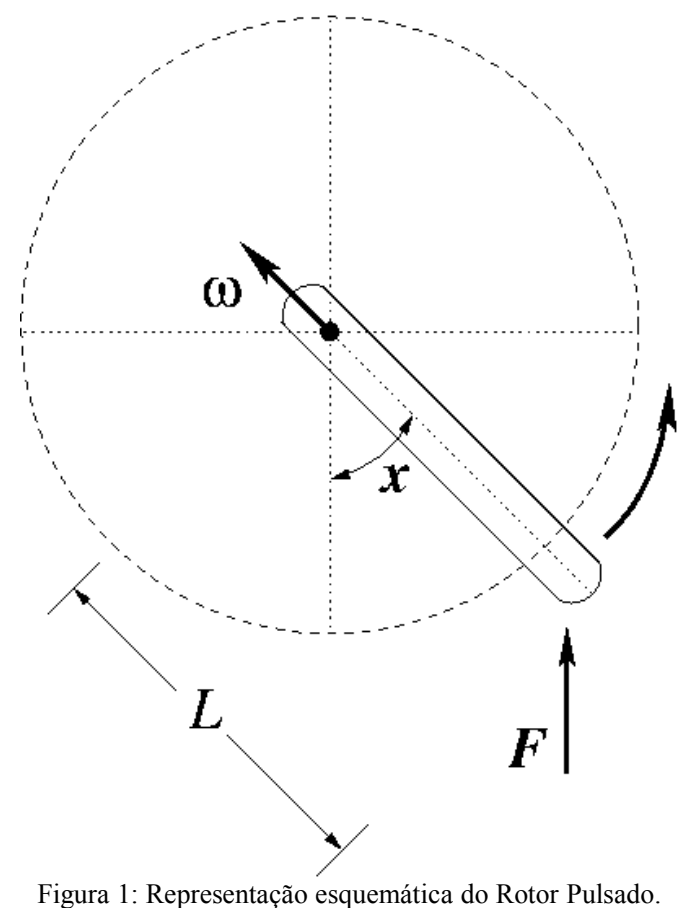

A função Hamiltoniana que descreve a dinâmica do rotor pulsado é a seguinte:

$$
H(p, x, t)=\frac{p^{9}}{9 \mathrm{I}}+K \cos (x) \sum_{n=0}^{\infty} \delta(t-n \tau),
$$

sendo que $p$ e $x$ representam o momento e a posição angulares e $\delta(t-n \tau)$ é a função delta de Dirac. Assim, para $t=n \tau$, teremos $\delta(t-n \tau)=1$ e o sistema encontra-se sob a influência da presença de uma força externa (pulso periódico), porém se $t \neq n \tau$, teremos $\delta(t-n \tau)=0$ e a força externa sobre o sistema será nula. O primeiro termo da função Hamiltoniana representa a energia cinética e o segundo energia potencial do rotor pulsado. As equações de movimento deste sistema são dadas pelas seguintes equações canônicas (Lemos, 2007) :

$$
\begin{gathered}
\frac{d x}{d t}=x=\frac{\partial H}{\partial p}, \\
\frac{d p}{d t}=p=\frac{-\partial H}{\partial x},
\end{gathered}
$$

assim teremos:

$$
\begin{gathered}
\frac{d x}{d t}=\frac{p}{I}, \\
\frac{d p}{d t}=K \operatorname{sen}(x) \sum_{n=0}^{\infty} \delta(t-n \tau) .
\end{gathered}
$$

Para chegarmos ao mapa padrão, algumas considerações e mudanças de variáveis foram feitas, de acordo com Woellner, (2006), sendo que a partir daqui chamaremos de $y$ a variável relacionada ao momento angular $\mathrm{p}$ das equações de movimento acima (4) e (5). Deste modo, pode-se obter então o seguinte mapa (Lichtenberg e Lieberman, 1992):

$$
\begin{array}{cc}
y_{n+\ell}=y_{n}+\frac{K}{9 \pi} \operatorname{sen}\left(9 \pi \mathrm{x}_{n}\right) & (\bmod 1), \\
x_{n+\ell}=x_{n}+y_{n+\rho} & (\bmod 1),
\end{array}
$$

sendo $x$ e $y$ a coordenada espacial e o momento canonicamente conjugado, respectivamente, $n$ a variável temporal discreta e $K$ o parâmetro de não-linearidade, responsável pelo nascimento da dinâmica caótica (Manchein e Beims, 2013).

Iniciamos o estudo numérico da dinâmica do mapa padrão, apresentando na Fig. 2, o seu espaço de fases para $K=2,5$; construído com 100 condições iniciais (CIs), escolhidas aleatoriamente, no domínio $(x, y)=[0,0 ; 1,0] \mathrm{X}[-0,5 ; 0,5]$ e iteradas até $n=1000$. As CIs foram escolhidas de forma arbitrária, com o objetivo de respondermos a seguinte pergunta: como se organizam os domínios de dinâmica regular imersos no mar caótico para um valor de $K$ típico? Pode-se encontrar informações detalhadas a respeito da influência do parâmetro $K$ e dos regimes regulares e caóticos apresentados pelo mapa padrão em Manchein e Beims (2013). Em tal referência, mostramos como ocorre o nascimento da dinâmica caótica, no mapa padrão, através de diagramas de bifurcações construídos utilizando-se os expoentes de Lyapunov a tempofinito. Voltando a discussão da Fig. 2, ela poderia ser gerada para outros valores de tempo, porém a única diferença será o aumento no número de pontos no espaço de fases. Além disso, podemos visualizar a existência de domínios, cuja dinâmica é regular, chamados de ilhas de regularidade (onde não há movimento caótico (Manchein e Beims, 2013), imersos no mar de caos (representados pelos pontos vermelhos). Em outras palavras, podemos observar uma região de caos (hachuras pontilhadas) e em torno do ponto fixo periódico em $(x, y)=(0,5 ; 0,0)$, chamado de ressonância (Lichtenberg e Lieberman, 1992). Em torno deste ponto existe uma considerável região de estabilidade formando a ilha primária cercada por outras quatro ilhas menores. Se iterarmos o mapa padrão por um intervalo de tempo maior, aumentando o número de pontos vermelhos no espaço de fases, podemos realizar ampliações das ilhas menores e perceber que existem várias outras ilhas cada vez menores em torno da ilha ampliada. Este padrão se repete indefinidamente, constituindo a característica autossimilar ou tipo-fractal, inerente aos sistemas deste tipo. As ilhas de regularidade são delimitadas por linhas, denominadas órbitas quase-periódicas, em que a cada iterada o estado do sistema pode ser representado por um 
ponto muito próximo ao anterior, formando uma linha aparentemente contínua. Analisando o interior das ilhas formadas no espaço de fases, podemos notar que existem várias órbitas quase-periódicas: cada uma delas gerada a partir de um par de CIs de $(x, y)$. Se usássemos um conjunto maior de condições iniciais, o número de órbitas quase-periódicas seria ainda maior, e eventualmente, preencheria quase-completamente o interior da ilha principal. Em outras palavras, as regiões brancas localizadas próximo ao centro da ilha e ao redor dela, representam pontos em que nenhuma condição inicial fora escolhida.

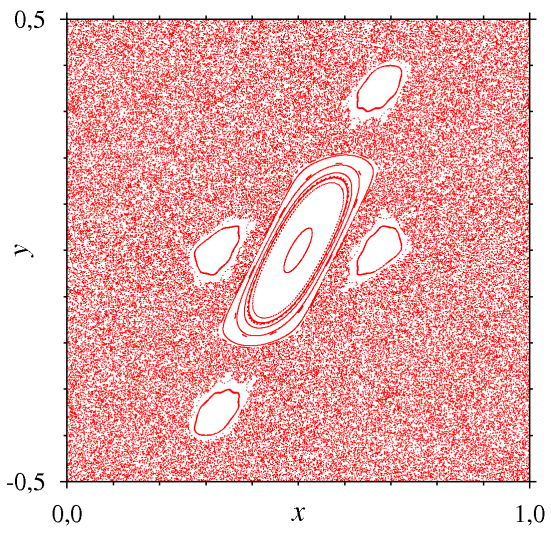

Figura 2: Espaço de fases do mapa padrão, para $\mathrm{K}=2,5$.

Por outro lado, o papel da amplitude do forçamento, representado pelo parâmetro $K$, refere-se a alterar o tamanho e quantidade de domínios de dinâmica regular e caótica. Este parâmetro pode ser alterado desde zero até valores muito grandes. No limite de $K \rightarrow 0$ o espaço de fases do mapa padrão é repleto de órbitas quase-periódicas, ou seja, o sistema não apresenta regimes caóticos no espaço de fases (Manchein e Beims, 2013).

Neste caso, para verificarmos a influência do parâmetro de não-linearidade na dinâmica do mapa padrão conservativo foram construídas três imagens (Fig. 3) para três valores diferentes de $K$. Podemos observar na Fig. 3(a), onde consideramos um forçamento relativamente pequeno $(K=0,5)$, a existência de trajetórias quase-periódicas e a inexistência de domínios caóticos. Aumentando a amplitude da força externa, para $K=4$, na Fig. 3(b), vê-se a presença dominante de caos e uma ilha de regularidade composta por trajetórias quase-periódicas, localizada no centro do espaço de fases. Na Fig. 3(c), para $K=5$, existe uma ilha que foi "quebrada" em duas ilhas menores, aumentando o tamanho da região caótica. Portanto, conclui-se que, nestes três casos quanto maior for o valor de $K$ "mais caótico" é o sistema. É importante ressaltar que, eventualmente pode-se escolher valores de $K$, em que o padrão detectado para estes três casos não exista.
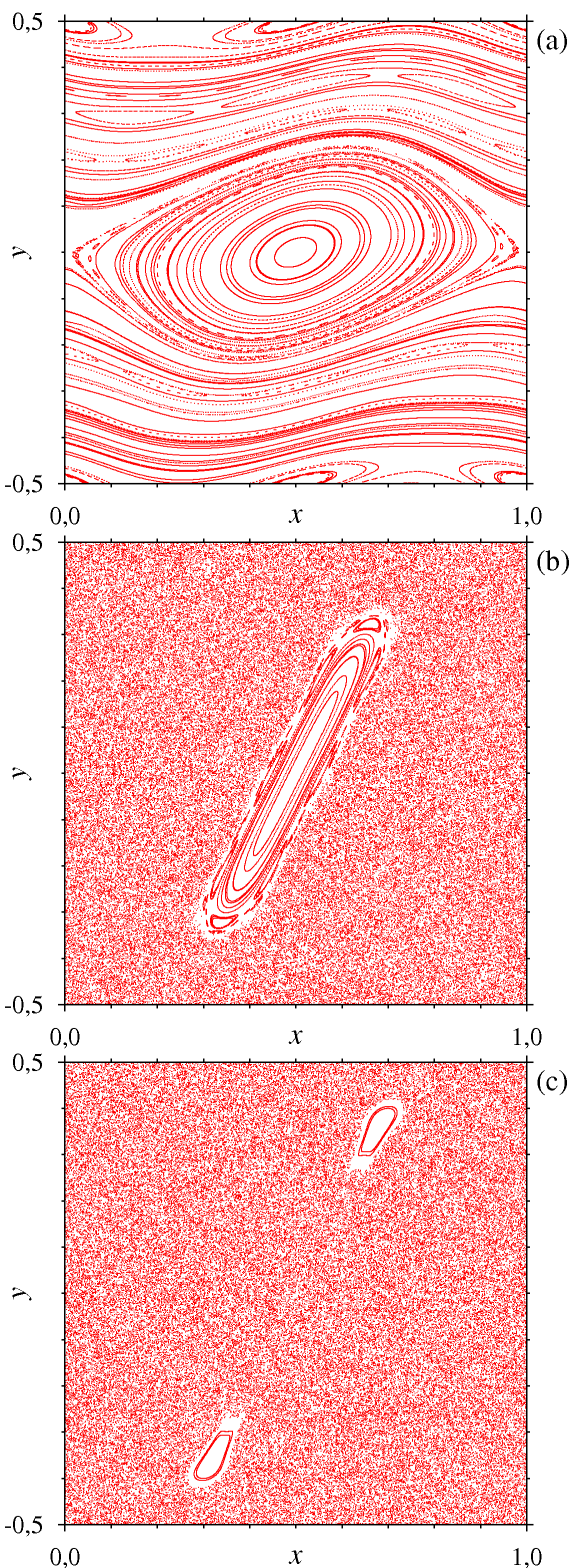

Figura 3: Espaço de fases do mapa padrão, para (a) $K=0,5$; (b) $K=4$ e (c) $K=5$. Estas imagens foram geradas com 100 condições iniciais escolhidas aleatoriamente e iteradas 1000 vezes cada.

\section{Mapa Padrão Dissipativo}

Geralmente em sistemas Físicos, existe pelo menos uma pequena quantidade de amortecimento ou dissipação. Por este motivo, faz-se interessante considerar termos dissipativos em modelos - até então conservativos - utilizados para descrever problemas físicos mais realísticos. Para estudarmos a influência da dissipação na dinâmica do mapa padrão, consideraremos a versão deste sistema estudado por Wenzel et al., (1991). Neste trabalho, os autores apresentam resultados referentes ao mapa padrão dissipativo, para o regime caótico e não-caótico, utilizando uma técnica numérica que permitiu-lhes encontrar órbitas periódicas estáveis e instáveis. 
Neste contexto, o rotor pulsado submetido a uma pequena quantidade de amortecimento $B$ pode ser descrito pelo seguinte mapa:

$$
\begin{array}{ll}
y_{n+1}=B y_{n}+\frac{K}{2 \pi} \operatorname{sen}\left(2 \pi \mathrm{x}_{n}\right) & (\bmod 1), \\
x_{n+1}=x_{n}+y_{n+1} & (\bmod 1),
\end{array}
$$

em que $x, y$ e $K$, têm o mesmo significado físico descrito nas Eqs. (6) e (7) e $B$ representa a dissipação que foi introduzida no sistema. Para $B=1$, a energia do sistema é conservada e para $B=0$, o sistema estará no limite super-dissipativo. $O$ espaço de fases do mapa padrão dissipativo pode ser analisado na Fig. 4, mantendo fixo $K=2,5$. Quando a dissipação for considerada, ela destrói as órbitas quase-periódicas, fazendo aparecer atratores no espaço de fases. A Fig. 4(a) representa o sistema no limite conservativo, ou seja, sem dissipação (compare com a Fig. 2). Nas imagens sequenciais aumentamos gradativamente a dissipação de $B=0,99999$ na Fig. 4(b) até $B=0,1$ na Fig. 4(f). Na Fig. 4(b) observa-se que as órbitas quase-periódicas estão mais espessas devido ao incremento do parâmetro $B$ com relação a Fig. 4(a). Nas Figs. 4(c-f), podemos constatar a quebra total das órbitas e a formação de atratores, ocorrendo uma brusca mudança estrutural do espaço de fases. Portanto concluímos que, a medida que a dissipação aumenta, as órbitas quaseperiódicas são quebradas ocasionando o aparecimento de atratores no espaço de fases.

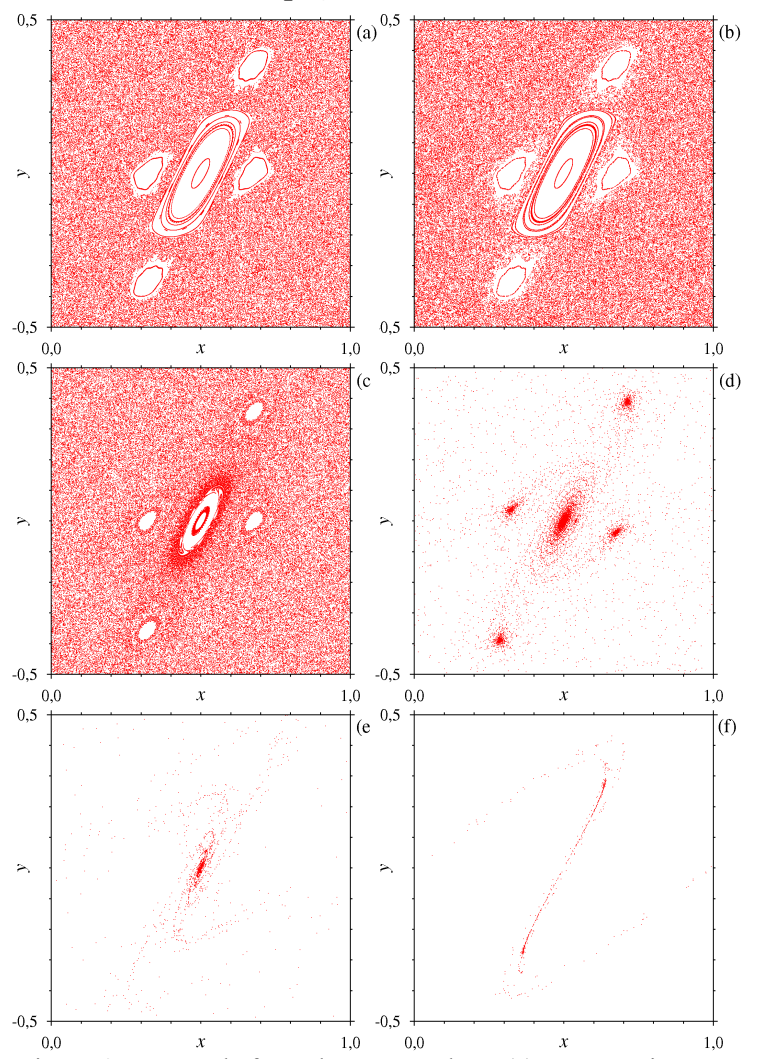

Figura 4: Espaço de fases do mapa padrão: (a) conservativo para $B=1$; e dissipativo para (b) $B=0,99999$; (c) $B=0,999$; (d) $B=0,9$ (e) $B=0,5$; (f) $B=0,1$. Todas as figuras foram geradas para $K=2,5$ e 100 condições iniciais escolhidas aleatoriamente e iteradas 1000 vezes cada.

\section{Mapa Padrão Dissipativo Relativístico}

Nesta seção, analisaremos o comportamento de uma partícula relativística, na presença de uma pequena quantidade de dissipação. Tal partícula é denominada relativística, pois pode apresentar velocidades comparáveis a velocidade da luz no vácuo. O sistema conhecido por mapa padrão relativístico (MPR), pode ser obtido a partir de uma função Hamiltoniana, que descreve a dinâmica de um uma partícula relativística num pacote de onda, conforme proposto inicialmente por Chernikov et al. (1989).

Consideramos então o problema de uma partícula em um pacote de onda sob a ação de um campo eléctrico. Neste problema podem existir casos em que o movimento da partícula é tal que as equações de Newton possam ser aplicadas, para velocidades baixas da partícula, ou ainda o caso em que a aceleração das partículas é suficientemente grande, tal que as equações de Newton não possam mais ser aplicadas e as equações relativísticas devam ser levadas em conta. O mapa padrão relativístico pode ser definido considerando-se uma partícula em um pacote de ondas, em que o campo elétrico $E(x, t)$ é descrito pela seguinte equação:

$$
E(x, t)=\sum_{n=-\infty}^{\infty} E_{n} \operatorname{sen}\left(k_{n} x-\omega_{n} t\right),
$$

sendo $E_{n}$ a amplitude do número de componentes de Fourier da onda de campo eléctrico, $k_{n}$ o número de onda, $\omega_{n}$ a frequência angular e $n$ o índice de cada onda. Supondo que o pacote de ondas tem um espectro largo de tal forma que se possa dizer que $E_{n}=E_{0}$, $k_{n}=k_{0}$ e $\omega_{n}=n \omega$, conforme feito por Oliveira et al., (2012), teremos:

$$
E(x, t)=E_{0} \operatorname{sen}\left(k_{0} x\right) \sum_{n=-\infty}^{\infty} \cos (n \omega t),
$$

substituindo a decomposição do período de Fourier pela função delta de Dirac chegaremos a seguinte expressão:

$$
E(x, t)=E_{0} \tau \operatorname{sen}\left(k_{0} x\right) \sum_{n=-\infty}^{\infty} \delta(t-n \tau),
$$

sendo $\tau=2 \pi / \omega$. Assim a Hamiltoniana que descreve o movimento de um elétron com massa de repouso $\mathrm{m}_{0} \mathrm{e}$ carga e, em um campo elétrico é:

$$
H(p, x, t)=\sqrt{p^{2} c^{2}+m_{0}^{2} c^{4}}-\frac{e E_{0} \tau}{k_{0}} \cos \left(k_{0} x\right) \sum_{n=-\infty}^{\infty} \delta(t-n \tau)
$$

sendo que $c$ representa a velocidade da luz, $p=m_{0} v[1$ $\left.(v / c)^{2}\right]^{-1 / 2}$ e $x$ representam o momento e a posição angulares relativísticos, respectivamente, e $\delta(t-n \tau)$ é a função delta de Dirac. O primeiro termo da Hamiltoniana representa a energia cinética e o segundo termo a energia potencial do sistema. As equações de movimento do sistema obtidas a partir da função Hamiltoniana, são dadas por:

$$
\dot{x}=\frac{p c^{2}}{\sqrt{p^{2} c^{2}+m_{0}^{2} c^{4}}},
$$




$$
\dot{p}=-e E_{0} \tau \operatorname{sen}\left(k_{0} x\right) \sum_{n=-\infty}^{\infty} \delta(t-n \tau)
$$

considerando, $x=\mathrm{k}_{0} x$ e introduzindo a variável auxi$\operatorname{liar} \beta=\omega / K c, y=k_{0} p / m_{0} \omega$. O efeito de amortecimento ou dissipação pode ser considerado introduzindo-se um termo fenomenológico no mapa padrão relativístico, conforme artigo de Ciubotariu et al., (2002). Deste modo, chegamos à expressão do mapa padrão dissipativo relativístico (MPDR) descrito pelas seguintes equações (Oliveira et al., 2011 e Oliveira et al., 2012):

$$
\begin{aligned}
& y_{n+1}=B y_{n}-\frac{K}{2 \pi} \sin \left(2 \pi \mathrm{x}_{n}\right) \quad(\bmod 1), \\
& x_{n+1}=x_{n}+y_{n+1}\left(1+\beta^{2} y_{n+1}^{2}\right)^{-1 / 2}(\bmod 1),
\end{aligned}
$$

O MPDR dado pelas Eqs. (16) e (17), possui três parâmetros que controlam: a amplitude $K$ dos pulsos recebidos pela partícula em intervalos discretos de tempo, a dissipação $B$ e o comportamento relativístico $\beta$. Para estudarmos a influência da componente relativística no sistema foram construídos espaços de fases para valores diferentes de $\beta$. Quando sistemas, cuja dinâmica da partícula seja modelada pelo MPDR apresentarem baixas velocidades, o que implica que o valor do parâmetro $\beta$ deva ser pequeno, não ocorrem grandes mudanças com relação a Fig. 4(a) discutida anteriormente (compare a Fig. 4(a) com a Fig. 5(a)). Em outras palavras, existem apenas pequenas ilhas no entorno das quatro ilhas menores. Porém aumentando o valor de $\beta$, observa-se uma diminuição gradativa da região caótica e o surgimento de vários domínios onde a dinâmica apresenta-se aparentemente regular, conforme pode ser visualizado nas Figs. 5(b-e) (outras investigações devem ser feitas nesta direção). A Fig. 5(f) ilustra o mapa padrão relativístico para $\beta=1$, ou seja, ocorre o caso de ressonância, com $K=2,5$ (força externa com amplitude relativamente pequena) e $B=1$ (ausência de dissipação).

Para analisar a influência da introdução de dissipação no MPDR, diminuímos gradativamente o valor do parâmetro $B$, indo em direção ao limite superamortecido. Partindo da imagem 5(d) reproduzida anteriormente, construímos uma sequência de imagens apresentadas a seguir. Assim como no mapa padrão, a componente relativa ao amortecimento no MPDR, também ocasiona o aparecimento de atratores.

Na Fig. 6(a) fixamos $\beta=0,6, K=2,5$, e $B=$ 0,999999 (dissipação pequena), onde não percebemos mudanças significativas, porém aumentando a dissipação sobre o sistema, Fig. 6(b) em diante, observamos o aparecimento de efeitos semelhantes aos detectados no mapa padrão dissipativo. Em outras palavras, nas figuras sequenciais, $6(\mathrm{c}-\mathrm{f})$, vemos claramente a formação de atratores. Assim como no caso do mapa padrão dissipativo, para o caso dissipativo relativístico, também temos a quebra das órbitas quase-periódicas, ocasionando o aparecimento de atratores no espaço de fases, conforme a dissipação fica mais intensa.
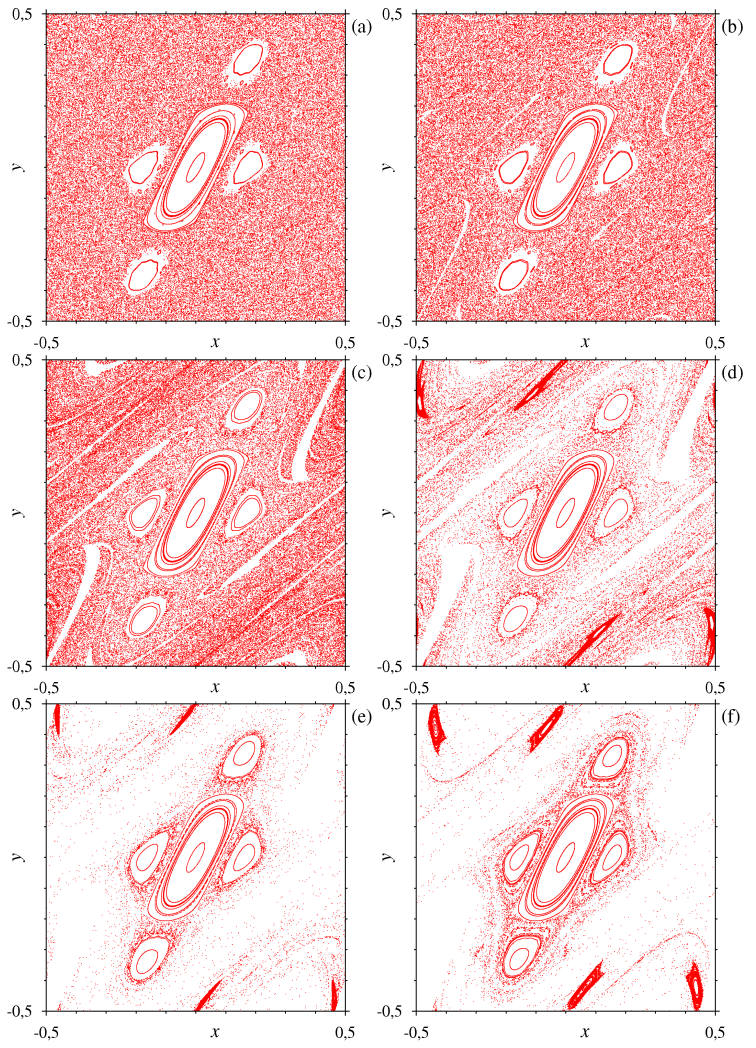

Figura 5: Espaço de fases do mapa padrão dissipativo relativístico: para (a) $\beta=0,1$; (b) $\beta=0,2$; (c) $\beta=0,4$; (d) $\beta=0,6$; (e) $\beta=0,8$; (f) $\beta=1$. Todas as figuras foram geradas para $B=1, \mathrm{~K}=2,5$ e 100 condições iniciais escolhidas aleatoriamente e 1000 iterações.
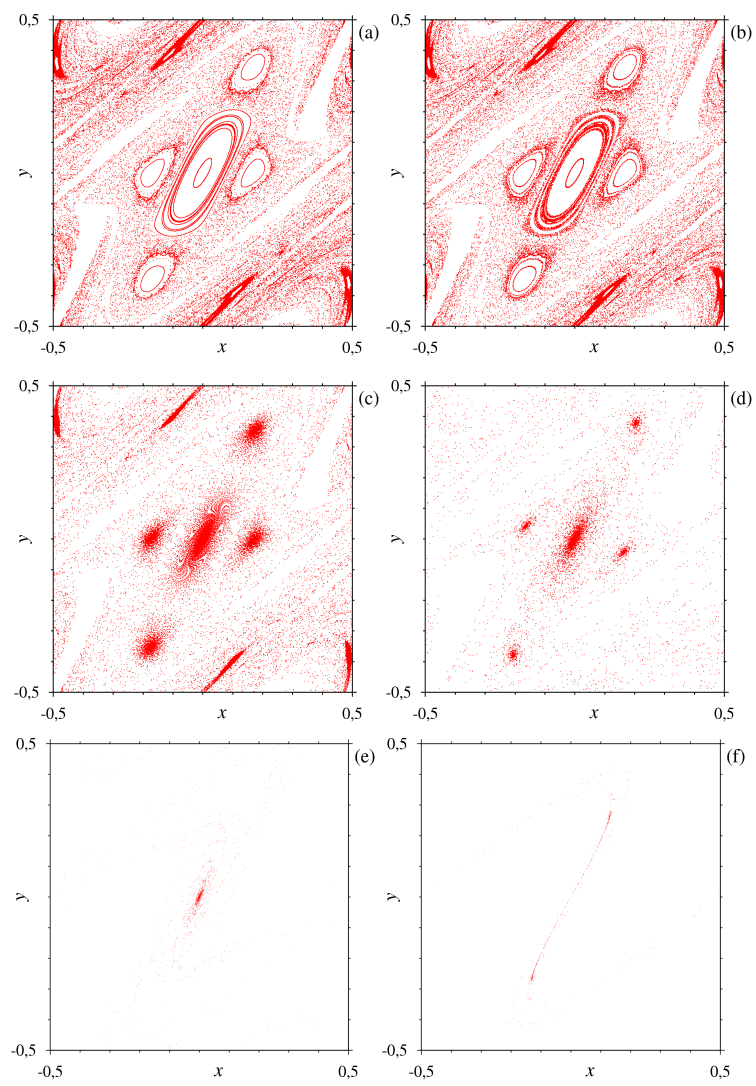

Figura 6: Espaco de fases do mapa padrão dissipativo relativístico: para (a) $B=0,999999$; (b) $B=0,9999$; (c) $B=0,99$; (d) $B=0,9$; (e) $B=0,5$; (f) $B=0,1$. Todas as figuras foram geradas para $\beta=0,6$; $K=2,5$ e 100 condições iniciais escolhidas aleatoriamente e 1000 iterações. 


\section{Conclusão}

Neste trabalho, estudamos a dinâmica do mapa padrão, no regime conservativo, dissipativo e ainda dissipativo relativístico, através de uma abordagem numérica. Os principais objetivos do mesmo referemse à compreensão e ao entendimento da dinâmica do mapa padrão nos regimes supracitados, e a caracterização da influência da variação do parâmetro relativístico na dinâmica do mapa padrão dissipativo relativístico. Com estes objetivos em mente, analisamos o comportamento de cada sistema através dos seus respectivos espaços de fases, à medida que aumentávamos o amortecimento e o parâmetro relativístico. Esperávamos como resultados, o surgimento de atratores no espaço de fases conforme aumentássemos a dissipação do sistema. Obtivemos exatamente este resultado: conforme aumentávamos o parâmetro de dissipação percebíamos que a estrutura do espaço de fases sofria grandes mudanças estruturais com o surgimento de vários atratores. Estes por sua vez surgiam devido a quebra das trajetórias quase-periódicas que delimitam as ilhas de regularidade, do caso onde a energia é uma quantidade conservada. O número de atratores aumentava conforme nos aproximávamos do limite conservativo em todos os casos estudados. Próximo a este limite podem existir milhares de atratores, conforme mostrado para o rotor pulsado no artigo de Martins e Gallas, (2008). Além disso, o incremento do parâmetro relativístico mostrou-se essencial no aparecimento de domínios de regularidade no espaço de fases no caso onde a energia é uma quantidade conservada.

\section{Agradecimentos}

ACCH agradece a CAPES (Coordenação de Aperfeiçoamento de Pessoal de Nível Superior) pela bolsa de estudos.

\section{Referências Bibliográficas}

Chernikov, A.A., Tél , T., Vatay, G., Zaslavsky, G.M., (1989) Chaos in the relativistic generalization of the standard maps, Phys. Rev. A, 40, 4072-4076. DOI: 10.1103/PhysRevA.40.4072

Chirikov, B.V., (1969) Research concerning the theory of nonlinear resonance and stochasticity, Institute of Nuclear Physics, Novosibirsk, Preprint No. 267.

Chirikov, B.V., (1979) Physics Reports 52263.

Ciubotariu, C., Bădeliță, L. e Stancu, V. (2002) Chaos in dissipative relativistic standard maps, Chaos, Solitons and Fractals 13, 1253-1267.

Lan, B. L. e Yapp, C.. (2008) Dissipative relativistic standard map: Periodic attractors and basins of attraction, Chaos, Solitons and Fractals 37, 1300-1304. DOI: 10.1016/j.chaos.2006.10.066
Lemos, N.A., (2007) Mecânica Analítica, 2 ed., Editora Livraria da Física.

Lichtenberg, A. J.; Lieberman, M. A. (1992) Regular and Chaotic Dynamics. New York: SpringerVerlag. DOI: 10.1007/978-1-4757-2184-3

Martins, L. C. e Gallas, J. A. C. (2008) Multistability, phase diagrams and statistical properties of the kicked rotor: a map with many coexisting attractors, International Journal of Bifurcation and Chaos 18, 1705-1717. DOI: 10.1142/S0218127408021294

Manchein, C.; Beims, M.W. (2013) Conservative Generalized Bifurcation Diagrams, Physics Letters A, v. 377, 789. DOI: 10.1016/j.physReta.2013.01.031

Oliveira, D. F.M., Leonel, E. D., Robnik, M. (2011) Boundary crisis and transient in a dissipative relativistic standard map, Physics Letters A 375, 3365-3369. DOI: 10.1016/j.physleta.2011.07.045

Oliveira, D. F.M., Leonel, E. D., Robnik, M. (2012) Dynamical properties of a particle in a wave packet: Scaling invariance and boundary crisis, Physics Letters A 376, 3630-3637. DOI: 10.1016/j.physleta.2012.10.052

Wenzel, W., Biham, O., Jayaprakash, C., (1991) Periodic orbits in the dissipative standard map, Phys. Rev. A, 43, 6550-6557. DOI: 10.1103/PhysRevA.43.6550

Woelner, C. F., (2006) Aspectos dinâmicos de uma rede de mapas Hamiltonianos acoplados, Dissertação de mestrado, UFPR. 